\title{
Immunohistochemical analysis of Musashi-1 expression during retinal regeneration of adult newt
}

\section{Jun Kaneko, Chikafumi Chiba*}

Graduate School of Life and Environmental Sciences, University of Tsukuba, Tennoudai 1-1-1, Tsukuba, Ibaraki 305-8572, Japan

* Corresponding author. Tel: +81 29853 4667; fax: +81 298536614.

E-mail address: chichiba@biol.tsukuba.ac.jp (C. Chiba).

URL: http://www.biol.tsukuba.ac.jp/ chichiba/

Both authors contributed equally to the paper.

Number of text pages of the whole manuscript: 13

Number of figures: 3

\section{Acknowledgements:}

This work was supported by Grant-in-Aid for Scientific Research (C) and Grant-in-Aid for Exploratory Research from the Japan Society for the Promotion of Science. We thank Dr. Hideyuki Okano for giving us the $14 \mathrm{H} 1$ antibody.

Keywords: Musashi-1; regeneration; retina; retinal pigment epithelium; photoreceptor; newt 


\section{Abstract}

The adult newt retinal regeneration is an ideal model for studying retinal regeneration by transdifferentiation of the retinal pigment epithelium (RPE) cells. Accumulated evidence suggests that the RNA-binding protein Musashi-1 (Msi1) is expressed in mature photoreceptors and RPE cells as well as in retinal stem/progenitor cells, being essential for vision. We have been investigating whether Msi1 is also essential for retinal regeneration. In the last paper (Susaki et al., Exp. Eye Res., 2008), we showed that the expression profiles of Msi1 transcripts and protein isoforms change during retinal regeneration. In the current report, we show by immunohistochemistry that Msi1 is expressed in transdifferentiating cells or cells of regenerating retinal tissues. Upon retinectomy, Msi1 protein, which is expressed in the nuclei of intact (stage E-0) RPE cells, changed its subcellular localization, being expressed in both the nucleus and cytoplasm of the RPE-derived stem-like cells at stage E-1. As the retinal rudiment/regenerating retina (rR) and renewing RPE (rRPE) are specified from the stem-like cell population (stage E-2), Msi1 expression was maintained or up-regulated in the rR, while down-regulated in the rRPE. During further retinal regeneration, Msi1 expression was decreased in association with cell differentiation, except for photoreceptors and RPE cells whose Msi1 expression increased as they differentiate. Thus, Msi1 is likely to be regulated at various cellular events during retinal regeneration, implying that Msi1 may have multi-functions in retinal regeneration. All together, it is probable that Msi1 is one of the essential factors that needs to be regulated in retinal regeneration. 
Vision is one of the most important senses for humans. The retina is a part of the central nervous system (CNS) located in the eye, being essential for the initial process of vision. Therefore, diseases or traumatic injury of the retina seriously affect the normal life of humans. In contrast, some urodele amphibians such as newts can regenerate, even in adulthood, their entire retinas through the process of transdifferentiation (cell-type switch) of the retinal pigment epithelium (RPE) cells, even when the neural retina is completely lost (for review, see [6]). Recent advances in stem cell biology and regenerative medicine have revealed that, even in adult mammals including humans, RPE cells and cells in the pigmented ciliary margin and the iris pigment epithelium have the capacity to transdifferentiate into retinal cells in culture or after transplantation into the eyes [1, 3, 7, 11, 24]. As yet, however, we do not have a clinical strategy for the regeneration of the whole retina from pigment epithelium cells in the eyes of patients. Therefore, the retinal regeneration of adult newts can be a useful in vivo transdifferentiation model of the pigment epithelium cells to gain critical information aimed at establishing new clinical strategies for retinal regeneration.

Thus far, the retinal regeneration of adult newts has been studied by histological, biochemical and electrophysiological techniques [6]. When the neural retina is removed from the eye by surgery (retinectomy), RPE cells undergo a loss of pigment granules (depigmentation) and proliferation, and start generating a new retinal rudiment while renewing the RPE cell layer. Cells of the retinal rudiment continue to proliferate thereafter, producing retinal neurons and glia that eventually reform a new functional neural retina. Recently, we outlined the process of retinectomy-induced retinal regeneration as nine morphological stages $[5,6$, 16] (Fig. 1). However, the underlying cellular and molecular mechanisms remain largely unknown mainly because of technical limitations [6].

RNA-binding proteins (RBPs) are involved in a variety of processes that are critical for appropriate protein expression, for example, mRNA splicing, stability and localization, and translational control (for review, see [8]). Musashi is one of the highly conserved RBPs, and this family characteristically has two tandem RNA recognition motifs (RRMs) (for review, see [17]). Musashi was initially isolated in a fruit fly Drosophila melanogaster as a molecule required for asymmetric division of sensory organ precursor cells [15]; since then Musashi family genes have been identified in various animals including a roundworm Caenorhabditis elegans [26], a planarian 
Dugesia japonica [12], sea squirts Halocynthia roretzi and Ciona intestinalis [14], a newt Cynops pyrrhogaster [23], a frog Xenopus laevis [9, 19], a chick Gallus gallus [25], a mouse Mus musculus [20, 21], and human Homo sapiens [10].

In vertebrates, two family members, Msi1 and Msi2, have been identified. Msi1 is known to be expressed in stem-cells in the CNS, intestinal epithelium, mammary and germ lines, and has been postulated to play important roles in the maintenance of the stem-cell state, differentiation, and tumorigenesis [17]. In the mouse, Msi2 is known to be expressed in neural stem-cells and subsets of differentiated neurons in the CNS [21]. Msi2 has been postulated to act as Msi1 in regulating neural stem-cells [17, 22], but its roles in differentiated neurons remain unknown.

RNA targets of Msi1 and Msi2 remain largely unknown. However, in mammals, one target of Msi1 has been identified as Numb mRNA whose protein inhibits the activation of Notch-1 signaling pathway which positively regulates neural stem-cell self-renewal [17]. Another one is a cyclin-dependent kinase inhibitor p21 ${ }^{\text {WAF-1 }}$ mRNA [4]. It has been demonstrated that Msi1 represses translation of Numb and $p 21^{\text {WAF-1, }}$ regulating cell cycle and differentiation [4, 17].

Accumulating evidence suggests that Msi1 is also expressed in differentiated photoreceptors and RPE cells in adult eyes. The expression of Msi1 in photoreceptors was first reported in the fruit fly D. melanogaster [13]. Also in the larval frog X. laevis, it was reported that post-mitotic photoreceptors and RPE cells continue to express Msi1 [2]. Recently, we reported that Msi1 is expressed in mature photoreceptors and RPE cells in the adult newt C. pyrrhogaster [23]. Also in adult mice, Raji et al. [18] and we [23] reported that almost all cells, including the photoreceptors and RPE cells in the ocular tissues, express Msi1. Furthermore, we demonstrated using the adult newt retinal regeneration system that the expression profiles (Msi1a < Msi1d) of the Msi1 transcripts and protein isoforms in the photoreceptors are different from those (Msi1a > Msi1d) in the retinal stem/progenitor cells, and using the Msi1 knockout (Msi1-KO) mouse system that has defects in Msi1 results in the degeneration of photoreceptors (apoptosis of cones and rods and fragmentation of rod outer segments) and lack of a visual cycle protein RPE65 in the microvilli of RPE cells [23]. These observations suggest that Msi1 is essential for vision. Analyses on the target RNAs and roles of Msi1 in those differentiated cells have just begun.

Since Msi1 is expressed in mature photoreceptors and RPE cells as well as in 
retinal stem/progenitor cells, it is reasonable to hypothesize that Msi1 should also play important roles in retinal regeneration through transdifferentiation of RPE cells. In our last paper [23], we showed by RT-PCR and western blot analyses that the expression profiles of the Msi1 transcripts and protein isoforms change during retinal regeneration of the adult newt (see Fig. 3 in [23]); the ratio of Msi1a to Msi1d increases upon retinectomy, reaches a maximum at 19-days post-operation (po) when the regenerating retina becomes up to 3-4 cells thick, and then decreases gradually to reach low levels at 45-days po when almost all cells have exited the cell-cycle and differentiated functionally. However, to gain a better understanding of such changes in the expression profiles of Msi1 during retinal regeneration, we have to investigate what types of cells express Msi1 in regenerating retinal tissues. Therefore, in this short report, as an extension of our preceding paper and fundamentals for future studies on the retinal regeneration, we show spatial expression patterns of Msi1 in regenerating retinas at different morphological stages (Fig. 1) by means of immunohistochemistry with a monoclonal antibody $14 \mathrm{H} 1$ which recognizes an epitope (LAPGYTYQFP) in all the newt Msi1 isoforms [23].

Here we used the adult newt, C. pyrrhogaster $(11-12 \mathrm{~cm})$. Retinectomy and immunohistochemistry were performed as described previously [23]. The primary antibody 14H1 (rat monoclonal anti-Msi1 antibody, 1:200) was kindly provided by Dr. Hideyuki Okano (Keio University School of Medicine, Japan). Immunoblot analyses in our last paper [23] reveal that 14H1 clearly detects Msi1b/Msi1c ( 35 kD) and Msi1d $(\sim 37 \mathrm{kD})$ in the normal neural retina, and Msi1a ( 39 kD) and Msi1b/Msi1c in regenerating retinal tissues at 19-days po. The secondary antibody was biotinylated goat anti-rat IgG antibody (1:500; Vector, Burlingame, CA, USA). The reactions were visualized with the ABC-DAB protocol (Vector). All protocols applied to this animal were approved by the University of Tsukuba Animal Use and Care Committee.

Immediately after retinectomy (day-0 po), most eyeballs are shrunken, but the RPE cells are found to be firmly attached to the basement membrane (Bruch's membrane) (stage E-0, Fig. 1). At this stage, 14H1-immunoreactivity (14H1-IR) was observed in the nuclei of RPE cells as in the intact eyes (data not shown; see Fig. 2 in [23]). Within the following 5-days, most RPE cells are swollen, and pigment granules diffuse in their cytoplasm (transitional stage between stage E-0 and E-1). Such RPE cells are mostly kept along Bruch’s membrane, but are occasionally detached from there. 
At this stage, 14H1-IR was located in all the RPE cell nuclei (Fig. 2A).

At 10-days po, RPE cells typically lie on the surface of each other to form a layer of a few cells in thickness (stage E-1, Fig. 1). These cells exhibit an oval or spindle shape in sections, while the density of the pigment granules is slightly decreased, and most of them have entered the cell-cycle by this stage. These cells are regarded as stem-like cells from which a new neural retina and RPE are generated [6]. Interestingly, at this stage, 14H1-IR was observed in the cytoplasm of those cells as well as in their nuclei, although the signal in the cytoplasm was lower than that in the nucleus (Fig. 2B).

At 14-days po, there is the first indication of the formation of a new retinal rudiment and RPE cell layer; partially de-pigmented cells form two distinct cell-layers (stage E-2, Fig. 1). At this stage, 14H1-IR was observed in both the cell-layers, but interestingly it was not uniform between them (Fig. 2C); the inner cell-layer facing the vitreous chamber, i.e., retinal rudiment/regenerating retina (rR), showed intense immunoreactivity, and the signal was observed in both the nucleus and cytoplasm, as observed at stage E-1; on the other hand, immunoreactivity in the outer cell-layer, i.e., renewing RPE (rRPE), apparently declined (arrows in Fig. 2C) when compared to that of stage E-1 (Fig. 2B).

At 19-days po, the RPE-derived $r R$ is found to be almost completely de-pigmented and becomes up to 3-4 cells thick (stage E-3 to I-1, Fig. 1). Cells in those regenerating retinas are known to express Notch-1 uniformly [16] and continue to proliferate actively [5]. On the other hand, the cells of rRPE obviously increase in pigmentation and exit the cell-cycle [5]. In these stages, intense and uniform 14H1-IR was observed in cells of the regenerating retinas (Fig. $3 \mathrm{~A}, \mathrm{~B}$ ). On the other hand, the cells of rRPE showed weak immunoreactivity (not shown) as observed at stage E-2 (Fig. 2C).

At 23-days po, regenerating retinas exhibit multiple layers of cells before the segregation of synaptic layers (stage I-2 to I-3, Fig. 1). In these stages certain types of retinal neurons such as the ganglion cells and photoreceptors differentiate [6]. At stage I-2, round- or oval-shaped cells appear along the most proximal margin of the regenerating retina (Fig. 1). These cells are known to express voltage-gated $\mathrm{Na}^{+}$ channels and show glutamate-like immunoreactivity, being probably premature ganglion cells. At this stage, such cells along the most proximal margin showed slightly decreased 14H1-IR (arrowheads in Fig. 3C), while most other cells in the regenerating 
retinas showed intense immunoreactivity. At stage I-3, premature photoreceptors which express rhodopsin or RB-1 antigen appear solitary or in clusters along the most distal margin of regenerating retinas, and some types of amacrin cells and horizontal cells also start differentiation. Interestingly, at this stage, relatively high 14H1-IR was recognized in premature photoreceptors at the most distal region (arrows in Fig. 3D), while other regions of regenerating retinas showed slightly decreased immunoreactivity.

At 28-days po, the outer and inner plexiform layers or synaptic layers are about to segregate (transitional stage between stage I-3 and L-1, Fig. 1), and premature bipolar cells appear in the nuclear layer between the two plexiform layers, i.e., in the presumptive inner nuclear layer [6]. At this stage, photoreceptors with high 14H1-IR lined along the most distal margin of the regenerating retinas (Fig. 3E and F). Interestingly, as shown in Fig. 3F, in advanced regenerating retinas at the same stage, a high level of immunoreactivity was maintained in some longitudinally elongated cells in the presumptive inner nuclear layer, while the immunoreactivity of other cells in the presumptive inner nuclear layer and cells in the presumptive ganglion cell layer apparently decreased. In addition, immunoreactivity in the cell nuclei of rRPE became recognizable again at this stage (red arrowhead and inset in Fig. 3F).

At 45-days po, regenerating retinas characteristically have two plexiform layers but those layers are still thinner or immature (stage I-1, Fig. 1). At this stage, 14H1-IR was observed in all photoreceptors along the outer nuclear layer and a small number of cells in the inner nuclear layer (Fig. 3G). As shown in Fig. 3g, the cells in the inner nuclear layer typically had an oval shaped soma which located near the inner plexiform layer (arrow) and extended a process toward the outer plexiform layer (arrowheads). Immunoreactivity was also observed in the cell nuclei of rRPE (red arrowhead and inset in Fig. 3G). Later than 65-days po (stage L-2, Fig. 1), the neural retina and RPE, and 14H1-IR were almost identical to those in the intact eyes (data not shown).

The adult newt retinal regeneration system can be an ideal model for studying retinal regeneration by transdifferentiation of RPE cells [6]. The current immunohistochemical study demonstrated that the RNA-binding protein Msi1, which is expressed in the nuclei of intact (stage E-0) RPE cells as well as in photoreceptors [23], is also expressed in transdifferentiating cells or cells of regenerating retinal tissues.

After retinectomy, RPE cells change their morphology and enter the S-phase of the cell-cycle, reaching stage E-1, within 10 days under our experimental conditions. 
During this period, RPE cells repress their phenotypic genes such as RPE65 and simultaneously express new regulatory genes such as Pax6, a retinal stem-cell marker, while preserving certain original characteristics such as the presence of melanin pigments and RPE65 protein [6]. PCR analysis results in our last paper [23] suggested that the expression profile of the Msi1 transcripts in the posterior eye-cups at 10-days po is similar to that in the retinal stem/progenitor cells: The amount of the longest variant of Msi1, Msi1a, is larger than that of the shortest one, Msi1d. Immunohistochemical observations in the current report suggest that cells which express Msi1 in the posterior half of the eyeball at 10-days po are the RPE cells. Interestingly 14H1-IR was observed not only in the nucleus but also the cytoplasm of RPE cells, suggesting that Msi1 protein changes its subcellular localization at stage E-1. In stem/progenitor cells, Msi1 is known to be involved in translational regulation in the cytoplasm [4, 17]. Therefore, this change may reflect the ability of the RPE cell to acquire a stem-cell property. All together, these findings support our assumption that the RPE cells are reprogrammed to acquire stem-cell properties within 10 days after retinectomy, becoming stem-like cells [6]. It is interesting to examine whether the subcellular localization and function of Msi1 depend upon its protein isoform.

At 14-days po, partially de-pigmented cells form two distinct cell-layers, i.e., retinal rudiment/regenerating retina (rR) and renewing RPE (rRPE) (stage E-2). The rR at this stage is assumed to have a retinal progenitor property [6]. Interestingly, immunohistochemical observations in the current report suggest that the expression of Msi1 is maintained or up-regulated in rR but down-regulated in rRPE. In mammals, Msi1 is known to regulate cell-cycle and differentiation through translational repression of Numb (a Notch-1 signaling inhibitor) and $p 21^{\text {WAF-1 }}$ (a cyclin-dependent kinase inhibitor) [4, 17]. Therefore, there is a possibility that Msi1 might be involved in the maintenance of the cell-cycle in rR and in the differentiation of the RPE. In fact, cells of rR continue to proliferate thereafter, while cells of rRPE cease proliferation and increase pigmentation within the following 5 days, i.e., by 19-days po [5]. However, we must note that during retinal regeneration of the adult newt Notch-1 is not expressed until 19-days po (stage E-3), although the expression of other Notch homologues earlier than 19-days po remains possible [16].

The current immunohistochemical observations suggest that regenerating retinas up to 3-4 cells in thickness at 19-days po (stage E-3 to I-1) express Msi1 uniformly and 
that during further retinal regeneration Msi1 expression in the regenerating retina decreases in association with cell differentiation (except for photoreceptor differentiation). Such a pattern has also been observed in expression of Notch-1 [16] and a proliferating cell nuclear antigen (PCNA) [5]. These observations imply a possibility that Msi1 might be involved in the maintenance of neural stem/progenitor cells or in the regulation of the cell-cycle and differentiation during retinal regeneration of the adult newt. To clarify this possibility, double staining of Msi1 with Notch-1 or PCNA is necessary.

Photoreceptors in the adult newt normal eyes highly express Msi1d rather than Msi1a, although physiological functions of Msi1d in photoreceptors remain unknown [23]. PCR analysis results in our last paper [23] suggest that the amount of Msi1d starts to increase around 19-days po and continues to increase during further retinal regeneration. Consistently, immunohistochemical observations in the current report suggest that premature photoreceptors which appear solitary or in clusters at the most distal region of the regenerating retina at 23-days po express Msi1 and that the Msi1-expressing photoreceptors increase in number during further retinal regeneration. These findings suggest that Msi1d is an essential molecule for photoreceptors. It may be necessary to investigate the roles of Msi1d in differentiation and maintenance of photoreceptors as well as in their physiological function.

The current immunohistochemical observations suggest that Msi1 is re-expressed in RPE cells in association with their re-differentiation during retinal regeneration. Such a pattern of Msi1 protein expression seems to correlate with that of a visual cycle protein RPE65 expression which tends to decrease at 23-days po and recovers from around 28-days po [5]. This implies that Msi1 may have some important functions in differentiated RPE cells. In fact, the mouse Msi1 knockout (Msi1-KO) lacks RPE65 in the microvilli of RPE cells [23], although the underlying mechanism remains to be understood.

In conclusion, it is probable that Msi1 is one of the essential factors that needs to be regulated for retinal regeneration by transdifferentiation of RPE cells. Msi1 seems to have multi-functions in retinal regeneration. In future studies, to prove the functions of Msi1 implicated from the current immunohistochemical obsevations, it is necessary to identify the target RNAs of Msi1 at every event in retinal regeneration. 


\section{References}

[1] I. Ahmad, L. Tang, H. Pham, Identification of neural progenitors in the adult mammalian eye, Biochem. Biophys. Res. Comm. 270 (2000) 517-521.

[2] M.A. Amato, S. Boy, E. Arnault, M. Girard, A.D. Puppa, A. Sharif, M. Perron, Comparison of the expression patterns of five neural RNA binding proteins in the Xenopus retina. J. Comp. Neurol. 481 (2005) 331-339.

[3] K. Amemiya, M. Haruta, M. Takahashi, M. Kosaka, G. Eguchi, Adult human retinal pigment epithelial cells capable of differentiating into neurons. Biochem. Biophys. Res. Commun. 316 (2004) 1-5.

[4] C. Battelli, G.N. Nikopoulos, J.G. Mitchell, J.M. Verdi, The RNA-binding protein Musashi-1 regulates neural development through the translational repression of p21 ${ }^{\text {WAF-1 }}$. Mol. Cell. Neurosci. 31 (2006) 85-96.

[5] C. Chiba, A. Hoshino, K. Nakamura, K. Susaki, Y. Yamano, Y. Kaneko, O. Kuwata, F. Maruo, T. Saito, Visual cycle protein RPE65 persists in new retinal cells during retinal regeneration of adult newt. J. Comp. Neurol. 495 (2006) 391-407.

[6] C. Chiba, V.I. Mitashov, Cellular and molecular events in the adult newt retinal regeneration. In: C. Chiba (Ed.), Strategies for Retinal Tissue Repair and Regeneration in Vertebrates: from Fish to Human. Research Signpost, India, 2007, pp. 15-33.

[7] B.L.K. Coles, B. Angénieux, T. Inoue, K. Del Rio-Tsonis, J.R. Spence, R.R. McInnes, Y. Arsenijevic, D. van der Kooy, Facile isolation and the characterization of human retinal stem cells. Proc. Natl. Acad. Sci. USA. 101 (2004) 15772-15777.

[8] G. Dreyfuss, V.N. Kim, N. Kataoka, Messenger-RNA-binding proteins and the messages they carry. Nat. Rev. 3 (2002) 195-205.

[9] P.J. Good, M.L. Rebbert, I.B. Dawid, Three new members of the RNP protein family in Xenopus. Nucleic Acids Res. 21 (1993) 999-1006.

[10] P. Good, A. Yoda, S. Sakakibara, A. Yamamoto, T. Imai, H. Sawa, T. Ikeuchi, S. 
Tsuji, H. Satoh, H. Okano, The human Musashi homolog 1 (Msi1) gene encoding the homologue of Musashi/Nrp-1, a neural RNA-binding protein putatively expressed in CNS stem cells and neural progenitor cells. Genomics 52 (1998) 382-384.

[11] M. Haruta, M. Kosaka, Y. Kanegae, I. Saito, T. Inoue, R. Kageyama, A. Nishida, Y. Honda, M. Takahashi, Induction of photoreceptor-specific phenotypes in adult mammalian iris tissue. Nat. Neurosci. 4 (2001) 1163-1164.

[12] S. Higuchi, T. Hayashi, H. Tarui, O. Nishimura, K. Nishimura, N. Shibata, H. Sakamoto, K. Agata, Expression and functional analysis of musashi-like genes in planarian CNS regeneration. Mech. Dev. 125 (2008) 631-645.

[13] Y. Hirota, M. Okabe, T. Imai, M. Kurusu, A. Yamamoto, S. Miyao, M. Nakamura, K. Sawamoto, H. Okano, Musashi and Seven in absentia downregulate Tramtrack through distinct mechanisms in Drosophila eye development. Mech. Dev. 87 (1999) 93-101.

[14] T. Kawashima, A.R. Murakami, M. Ogasawara, K.J. Tanaka, R. Isoda, Y. Sasakura, T. Nishikata, H. Okano, K.W. Makabe, Expression patterns of musashi homologs of the ascidians, Halocynthia roretzi and Ciona intestinalis. Dev. Genes Evol. 210 (2000) 162-165.

[15] M. Nakamura, H. Okano, J. Blendy, C. Montell, MUSASHI, a neural RNA-binding protein required for Drosophila adult external sensory organ development. Neuron 13 (1994) 67-81.

[16] K. Nakamura, C. Chiba, Evidence for Notch signaling involvement in retinal regeneration of adult newt. Brain Res. 1136 (2007) 28-42.

[17] H. Okano, H. Kawahara, M. Toriya, K. Nakao, S. Shibata, T. Imai, Function of RNA-binding protein Musashi-1 in stem cells. Exp. Cell Res. 306 (2005) 349-356.

[18] B. Raji, A. Dansault, J. Leemput, G. de la Houssaye, V. Vieira, A. Kobetz, L. Arbogast, C. Masson, M. Menasche, M. Abitbol, The RNA-binding protein Musashi-1 is produced in the developing and adult mouse eye. Mol. Vis. 13 (2007) $1412-1427$. 
[19] K. Richter, P.J. Good, I.B. Dawid, A developmentally regulated, nervous system-specific gene in Xenopus encodes a putative RNA-binding protein. New Biol. 2 (1990) 556-565.

[20] S. Sakakibara, T. Imai, K. Hamaguchi, M. Okabe, J. Aruga, K. Nakajima, D. Yasutomi, T. Nagata, Y. Kurihara, S. Uesugi, T. Miyata, M. Ogawa, K. Mikoshiba, H. Okano, Mouse-Musashi-1, a neural RNA-binding protein highly enriched in the mammalian CNS stem cell. Dev. Biol. 176 (1996) 230-242.

[21] S. Sakakibara, Y. Nakamura, H. Satoh, H. Okano, RNA-binding protein Musashi2: developmentally regulated expression in neural precursor cells and subpopulation of neurons in mammalian CNS. J. Neurosci., 21 (2001) 8097-8107.

[22] S. Sakakibara, Y. Nakamura, H. Satoh, H. Okano, RNA-binding protein Musashi family: roles for CNS stem cells and a subpopulation of ependymal cells revealed by targeted disruption and antisense ablation. Proc. Natl. Acad. Sci. USA 99 (2002) 15194-15199.

[23] K. Susaki, J. Kaneko, Y. Yamano, K. Nakamura, W. Inami, T. Yoshikawa, Y. Ozawa, S. Shibata, O. Matsuzaki, H. Okano, C. Chiba, Musashi-1, an RNA-binding protein, is indispensable for survival of photoreceptors. Exp. Eye Res. (2008) (in press).

[24] V. Tropepe, B.L.K. Coles, B.J. Chiasson, D.J. Horsford, A.J. Elia, R.R. McInnes, D. van der Kooy, Retinal stem cells in the adult mammalian eye. Science 287 (2000) 2032-2036.

[25] J.M. Wilson, K. Sato, E.A.G. Chernoff, T.L. Belecky-Adams, Expression patterns of chick Musashi-1 in the developing nervous system. Gene Exp. Pat. 7 (2007) 817-825.

[26] A. Yoda, H. Sawa, H. Okano, MSI-1, a neural RNA-binding protein, is involved in male mating behaviour in Caenorhabditis elegans. Genes to Cells 5 (2000) 885-895. 


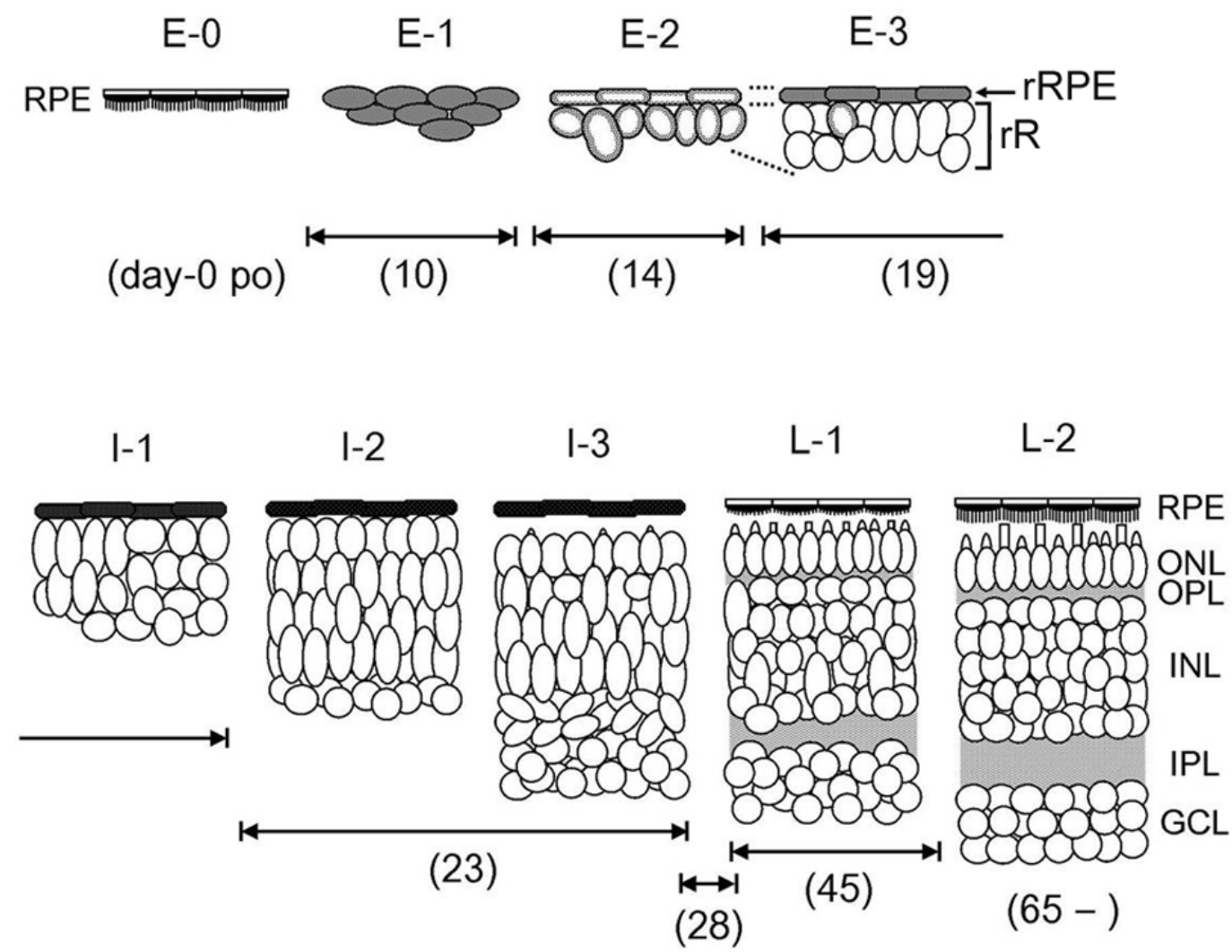

Fig. 1. Schematic diagram of adult newt retinal regeneration (modified from [16]). The process of retinal regeneration after retinectomy was divided into nine stages (E-0, E-1, E-2, E-3, I-1, I-2, I-3, L-1, L-2). The number in parenthesis indicates corresponding day post-operation (po) under the present experimental conditions. RPE, retinal pigment epithelium; rRPE, renewing RPE; rR, retinal rudiment/regenerating retina; ONL, outer nuclear layer; OPL, outer plexiform layer; INL, inner nuclear layer; IPL, inner plexiform layer; GCL, ganglion cell layer. For detailed explanations, see [6]. 

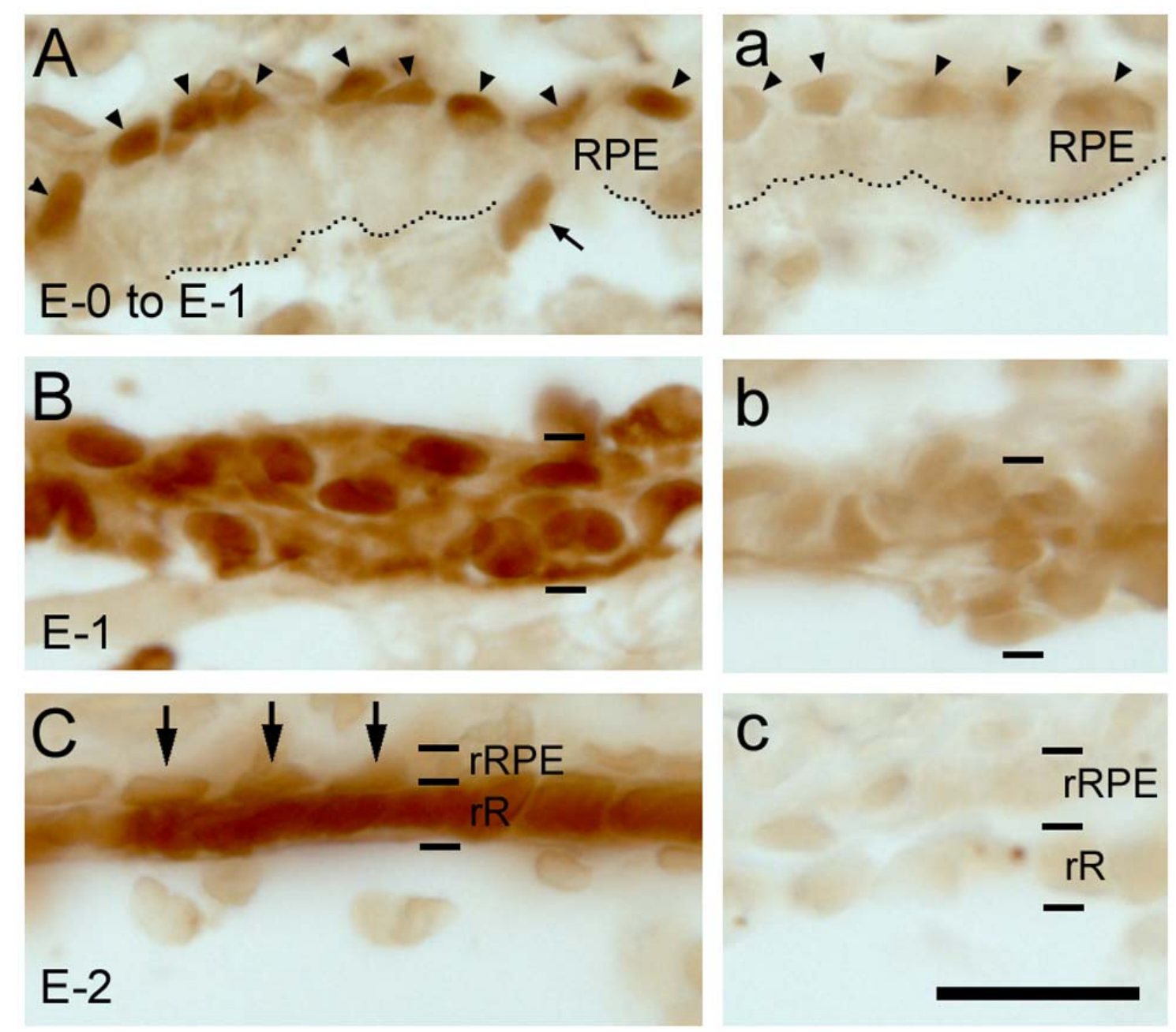

Fig. 2. 14H1-immunoreactivity in RPE cells at stage E-0 to E-1 (5-days po) (A, a), proliferating RPE cells (stem-like cells [6]) at stage E-1 (10-days po) (B, b), and retinal rudiment/regenerating retina (rR) and renewing RPE (rRPE) at stage E-2 (14-days po) $(\mathrm{C}, \mathrm{c})$. Right-hand panels (a, b, c) are the negative control without the primary antibody. Melanin pigments were bleached to view the immunoreactivity in pigmented cells. Arrowheads in (A) and (a): nuclei of RPE cells. Dotted lines in (A) and (a): vitreal ends of the microvili of RPE cells. Arrow in (A): the nucleus of an RPE cell detached from the basement membrane. Arrows in (C): nuclei of the rRPE. Scale bar= $50 \mu \mathrm{m}$. 

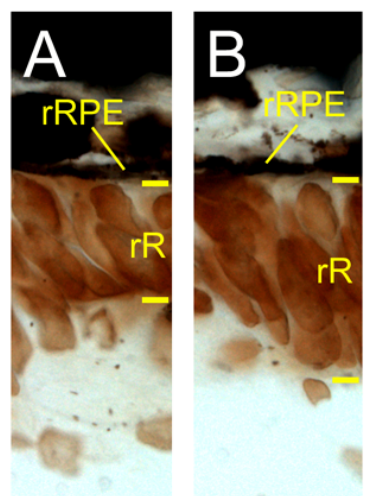

E-3

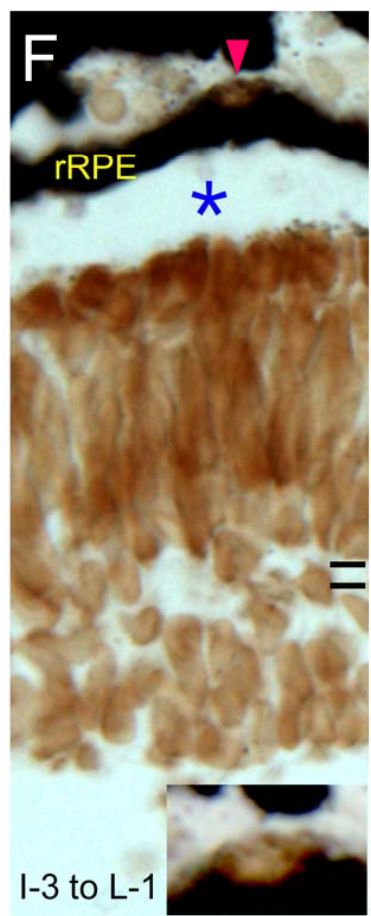

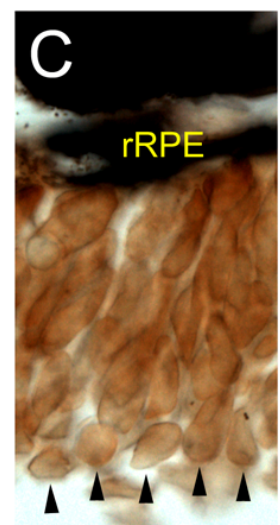

$\mathrm{I}-2$
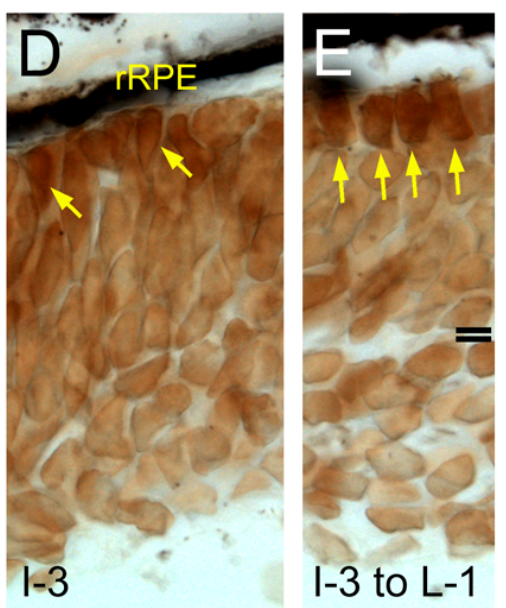

I-3 to L-1
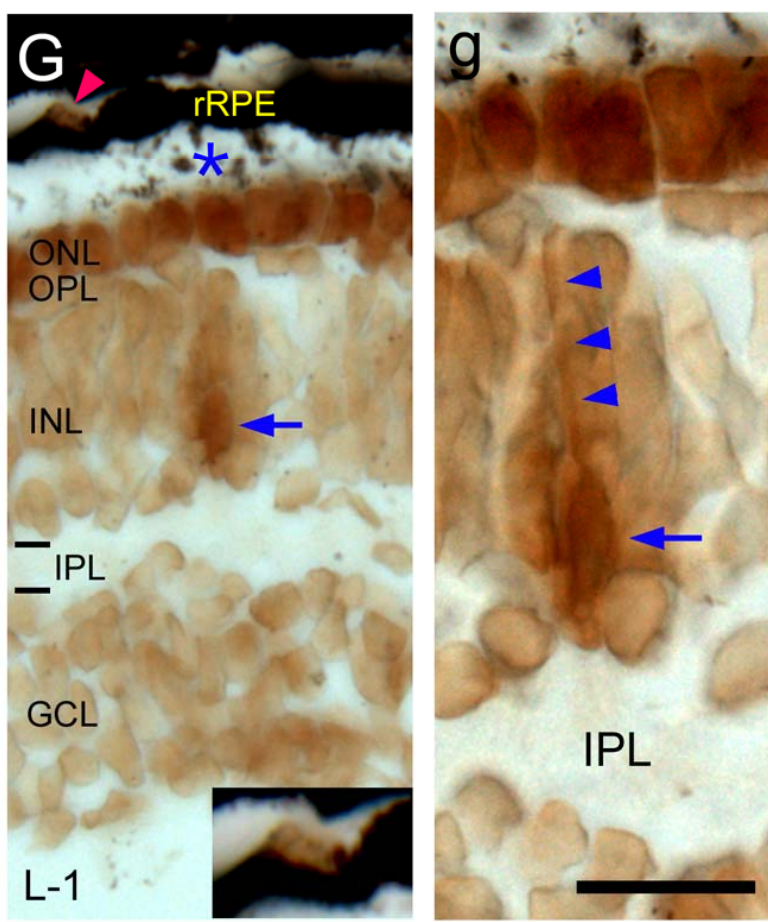

Fig. 3. 14H1-immunoreactivity in regenerating retinas (rR) and renewing RPE (rRPE) at stage E-3 (A) and I-1 (A) (19-days po), stage I-2 (C) and I-3 (D) (23-days po), transitional stage between I-3 and L-1 (E, F) (28 days po), and stage L-1 (G, g) (45days po). Arrowheads in (C): premature ganglion cells. Yellow arrows in (D) and (E): premature photoreceptors. Blue arrow in $(\mathrm{G})$ : a 14H1-positive cell remaining in the INL which is closed-up in (g). Blue arrowheads in (g): a process extending from the cell body. Red arrowheads in (F) and (G): 14H1-positive nuclei of rRPE cells which are closed-up in the insets at the bottom of the panels. Asterisks in (F) and (G): space caused by the detachment of rRPE from the regenerating retina during tissue sectioning. For abbreviations, see Fig. 1. Scale bar= $100 \mu \mathrm{m}$ (A-G); $50 \mu \mathrm{m}$ (g). 